\title{
Structural Changes and Gross Pathology of Gut and associated Organs of Mice on Chronic Exposure to Pan Masala Plain and its Blend (Pan Masala Tobacco): A Review and an Experimental Study
}

\author{
Suresh Kumar Nigam, Huthi Venkatakrishna Bhatt
}

\begin{abstract}
Swiss albino mice in three groups (control, experimental I and experimental II) of 60 each were fed staple diet (control), diet composed with $2 \%$ pan masala plain (PMP) and its blend (tobacco-PMT) for a period of up to 70 weeks with intermittent postmortem at 16, 56 and 70 weeks for assessing histological changes of the gut histopathology of mice. Mice of experimental groups I and II showed progressive advancement of structural changes of stomach during the study period. Fibrosis, thickening of prickle cells and also horney layered cells indicating lesions of acanthosis and hyperkeratosis initially and later prominently ulcers and papillomas which transformed into a peptic tumor occured. Spleen was found enlarged in mice fed with PMT. Animal feeding, hygiene, exploration and environmental care and health protocols were as per the standard guidelines and approved by National Institute of Occupational Health ethical committee. Here, chronic exposure of mice has been consistent in respect of feed and environment, unlike habitual PM users whose consumption is inconsistent depending on the body demand and choice on the variety of the blended PM product. This study endorses generating critical clinically designed case control, cohort data for awareness programs and PM substitutes as well as treatment to PM addicts.
\end{abstract}

Keywords: Pan masala, Gut changes, Mice.

How to cite this article: Nigam SK, Bhatt HV. Structural Changes and Gross Pathology of Gut and associated Organs of Mice on Chronic Exposure to Pan Masala Plain and its Blend (Pan Masala Tobacco): A Review and an Experimental Study. Euroasian J Hepato-Gastroenterol 2012;2(2):94-97.

Source of support: Nil

Conflict of interest: None

\section{INTRODUCTION}

Pan masala (PM) is a substance of dependence consumed like alcoholics, drug (opiates, cannabis, benzodiazepines) addicts. The PM is used as substitutes or adjuvants to psychoactive substances. Chronologically, over three decades several popular masticator products were composed, starting with areca nut, catechu, lime as betel quid, ${ }^{1}$ and added cardamom, permitted spices, unspecified flavoring, sweetening agents, etc. are sold as $\mathrm{PM}^{2}$ which further mixed with tobacco are known as 'Gutkha' or PMTobacco. ${ }^{3}$ To go partially inert to weariness of manual and nonmanual stress PM sachets are consumed occasionally to avoid halitosis and for relaxation and as mood elevator among working population. ${ }^{4}$ Thus, gradually dose-tolerance develops resulting in frequent (within 2-3 months) PM consumption and as a further reinforcement tobacco blended PM is consumed. ${ }^{5}$ Thus, PM addicts categorically show craving and substance seeking behavior (Guthka or Areca Nut Chewer's syndrome). ${ }^{6}$

The spurious blends of PM and high-flown advertisement and brisk marketing and their psychoactive stress ameliorating nature and usage by young and old as well as labor and executive class may immediately cater satisfaction; aphasia ensures zeal to do over work. ${ }^{7}$ To clear halitosis (freshen the breath), ${ }^{8}$ as analgesic, ${ }^{9}$ carminative, bactericidal, i.e. gastric digestion after a strong spicy food PM enables spontaneous strength from weariness. ${ }^{9,10}$ But over threshold limits and consistent and excessive consumption for long period results in functional and structural damage of organs, i.e. liver, ${ }^{8,11}$ lungs, testis, ${ }^{12}$ stomach, ${ }^{8}$ oral cavity, accessory organs, etc. and auditory, speech and visual deformities.

Fibrosis $^{13}$ is common in chronic PM, PMT chewers, beginning with inflammation ${ }^{14}$ progressive submucous fibrosis of oral mucosa, lungs, papillomas of stomach, liver, gut, testis, etc. The areca nut of PM has clastogenic, mutagenic, genotoxic and carcinogenic properties. The structural changes of gut on long-term exposure PM and its blend are hereby presented.

\section{MATERIALS AND METHODS}

Inbred Swiss mice with an average age of 6 weeks were set in three groups. Sixty animals each of both sexes in each group were exposed to PM (plain-PMP) and blended PM (with tobacco-PMT) and equal numbers of controls were provided with normal diet. Throughout the study period, pure inbred Swiss mice were used. The PMP, PMT fine powder in 100 gm quantity was mixed thoroughly with $4,900 \mathrm{gm}$ of feed given to mice routinely besides control animals which received only staple diet. The protocols of the experiments and maintenance of animals and their usage during this study were described in an earlier study. ${ }^{11}$

Exposed and control (Fig. 1) groups of animals were sacrificed by cervical dislocation in different time intervals. Postmortem in detail for gross pathology (Fig. 2) and for histology of liver, kidney, heart, spleen, stomach, esophagus 


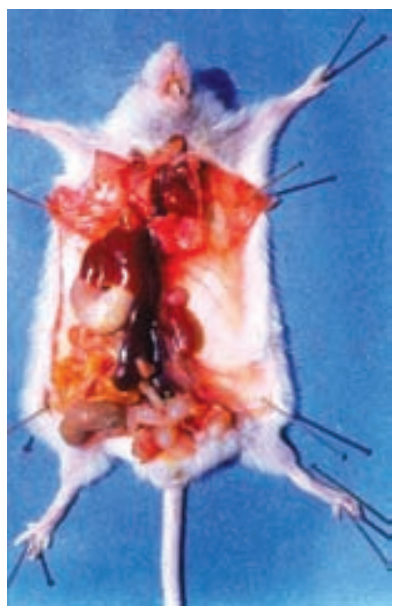

Fig. 1: Dissection showing normal part of the control mice

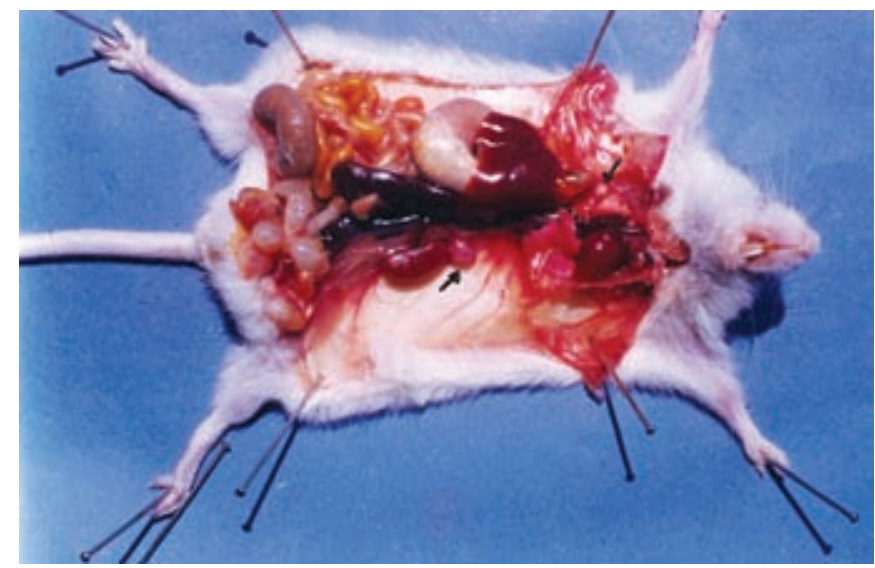

Fig. 2: Dissection showing (arrow) spleen and pathology of spleen in long-term PMT fed mice

and testis were collected. Organs were fixed in $10 \%$ buffered neutral formalin and processed routinely and $5 \mu \mathrm{m}$ thick paraffin sections were stained by hematoxylin and eosin. Tumor development was compared with control and evaluated by Students t-test.

\section{RESULTS}

The fore-stomach, apart the upper alimentary tract of the mouse, is apparently morphologically and physiologically akin to man. Fore-stomach of one animal fed with PMT (Gutkha) for 12 months showed mild thickening of prickle cells and also horny layer cells, thus indicating early hyperkeratosis (Fig. 3), lesions and squamous papilloma (Fig. 4) which is characterized by marked acanthosis, with infolding proliferation of squamous epithelium of the stomach (Fig. 5). Similarly liver exposed to sada as well as with Gutkha showed disrupted hepatocytes with fatty globular infiltration coalesced as fat cysts replacing total parenchyma and further reticular cell hyperplasia, hemangioma and hemangioendothelioma. Spleen and liver

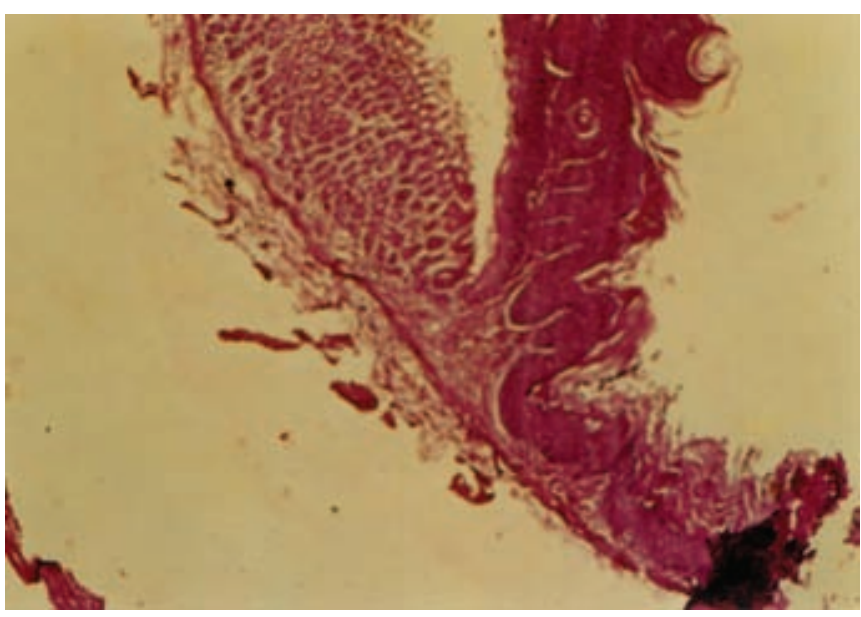

Fig. 3: PMT fed mice showing mild hyperkeratosis in the stomach

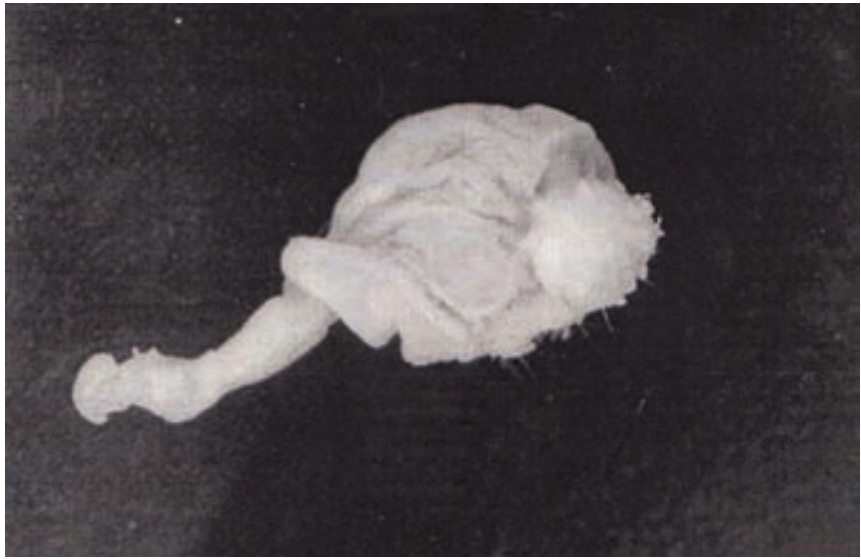

Fig. 4: Gross papilomatous growth (tumorous) dissected out from the stomach of mice fed with PMT

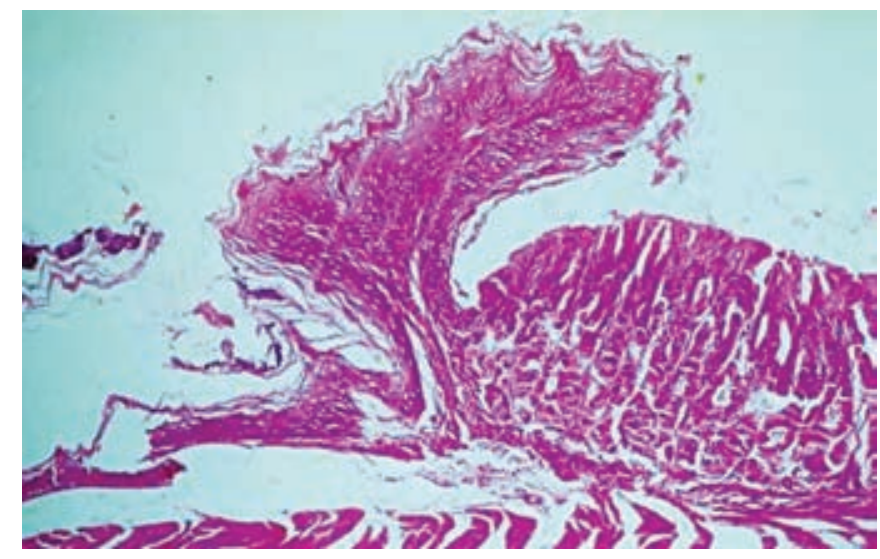

Fig. 5: Section of the gastric tumor in mice fed with PMT

were found enlarged (Figs 6 to 8) and damages other organs too, i.e. testes, lung and stomach in mice exposed to PMT.

\section{DISCUSSION}

In a preliminary report, we have shown that PMP and PMT longer time fed mice showed early biochemical changes and hepatocarcinogenesis. ${ }^{8-11}$ In the present study, analysis 


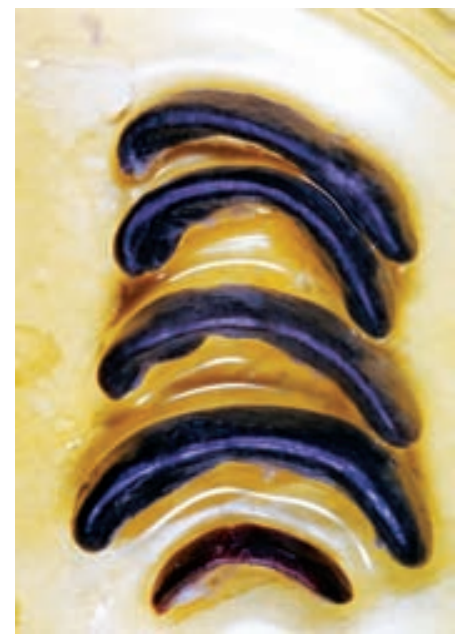

Fig 6: Extricated enlarged spleen of PMT fed mice

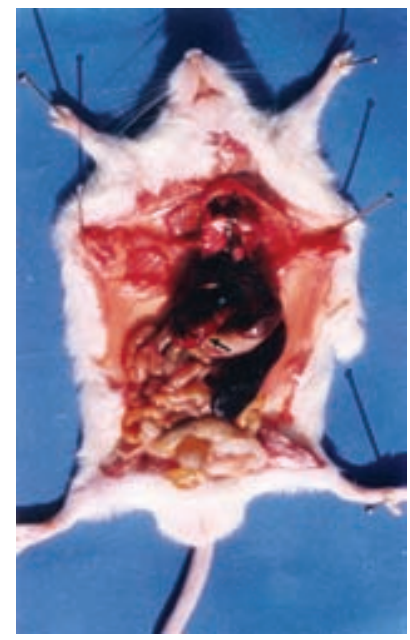

Fig. 7: Dissection showing (arrow) gross tumor and pathology of liver in long-term PM fed mice

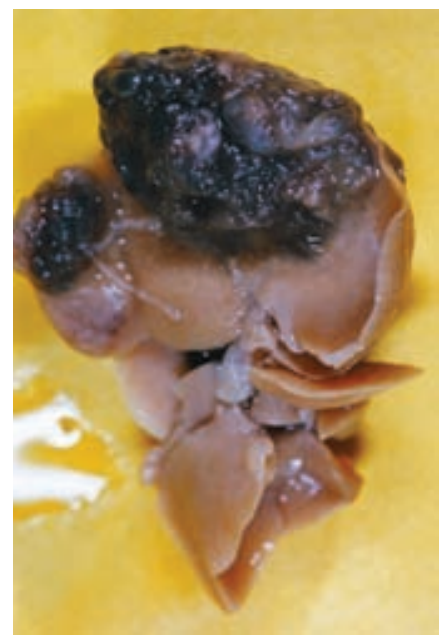

Fig. 8: Extracted liver showing gross tumor in chronic PMT fed mice of data of 2\% PM with or without tobacco fed Swiss mice for 70 weeks showed discrete and prominent structural changes not only in liver but also in stomach, lungs and reproductive organs. The histopathological changes concurrently advances with the gradual development of body burden due to PMP and further enhanced effect with PMT as shown in benzene hexachloride. ${ }^{15}$ The structural changes either dispersion due to infiltration of gut cells which show severe fatty changes (steatosis) as seen in PM exposure, may impair cellular function and can cause irreversible impairment of some intracellular process, such as decrease of protein synthesis, ${ }^{16,17}$ which is essential for conversion of triglycerides to lipoprotein excretion as seen in $\mathrm{CCl}_{4}$ poisoning. ${ }^{18}$

Carcinogenic potential of PM is referred to its contents, i.e. areca nut (alkaloids), catechu, polyaromatic hydrocarbons, nitrosomine ${ }^{19}$ toxic metals $(\mathrm{Pb}, \mathrm{Cd}, \mathrm{Ni})$, residual pesticides (DDT, BHC and their isomers) and fungi (Aspergillus species liberate afflatoxins which is a hepatocarcinogen ${ }^{20}$ ), Arecaidine (arecoline its methyl ester) promotes carcinogenesis, since it reacts with cysteine in vivo and in vitro to produce cysteine/3-alkylation adducts. ${ }^{21}$ The superoxide ions also initiate multiorgan carcinogenesis by way of autoxidation of polyphenols and interaction of catechin (tannin) with lime. ${ }^{19}$ Further, a case of liver cell carcinoma consisting of cords surrounded by sinusoidal cells with marked cell polymorphism was also seen in PM exposed group. This was explained to be an endogenous initiation of hepatocarcinogenesis in rodents and occurs by increasing the intracellular production of $\mathrm{H}_{2} \mathrm{O}_{2}$ which causes DNA strand breakage as well as chemical alterations of DNA bases. Damage to DNA is generally regarded as the initial biochemical alteration leading to neoplastic transformation in the case of the majority of chemical carcinogens which may be the prime factor even in this case for the induction of liver cancer. ${ }^{20}$ Carcinogenic activity was observed by pasting ethanol extract of PM on mice for 40 weeks which showed skin papillomas and enhanced its rate of conversion to carcinomas and at higher dose, promoted effectively the development of fore-stomach and esophageal papillomas and carcinomas. ${ }^{21,22}$ Prolonged usage of PMP, PMT in the feed of mice is a simulating condition to humans except dosage as PM user's quite often unlimited frequent consumption by chewing and swallowing the saliva and enables PM carcinogenic sites other than oral cavity, unlike tobacco chewers who often spit out the chewed stuff. In the present study, spleen and gastric damage (and to multiple organs) in mice, after chronic feeding of PM and gutkha (PMT) and even ultimately leading to gut cancer suggest that PM especially its blend may exert carcinogenic or even cocarcinogenic influence in man, specially in those who are habitual users of such products.

\section{ACKNOWLEDGMENTS}

The authors thank the Director, NIOH, Ahmedabad for facilities, support and encouragement during the period of study. One of the authors (Dr SK Nigam) further thank the Director-General, Indian Council of Medical Research, New Delhi for the award of EMS fellowship and funding during the study period. The authors also thank Miss Shagufta Shaik and Mr Ashok Kumar (Senior Research Officer, Animal House, NIOH, Ahmedabad) for the technical assistance during this study.

\section{REFERENCES}

1. Nigam SK, Bhatt HV. Chemical and experimental studies of plain and blended Indian pan masala. Proceeding of the National Academy of Sciences (India), 74th Annual Session, Jaipur, Section of Biological Sciences 2004 Dec 2-4:84. 
2. Nigam SK, Bhatt HV. Analysis and experimental evaluation of Indian blended pan masala. Proceedings of 94th Indian Science Congress (A), Annamalai University, Annamalai Nagar, Chidambaram, Tamil Nadu 2007 January;3-7.

3. Vatsala M, Premala AS, Nirupama L, Pooja A, Mamta S. Changing pattern of oral cavity lesions and personal habits over a decade: Hospital based record analysis from Allahabad. Indian J Comm Med 2009;34:321-25.

4. Srinivasan TN, Suresh TR, Vasantha J. Pan masala: A new substance of dependence. Indian J Psychiat 1993:35:189.

5. Nigam SK, Bhatt HV. Histopathology of chronic pan masala (PM) on the testis of mice. 76th Annual Session of National Academy of Sciences: Section of Biological Sciences (A:75, p:54), IIT\&TIFR, Mumbai 2006 Oct:6-8.

6. Chaturvedi P. Gutka or areca nut Chewer's syndrome. Indian J Cancer 2009:46:170-72.

7. Sushma C, Sharang C. Pan masala advertisements are surrogate for tobacco products. Indian J Cancer 2005;42:94-98.

8. Nigam SK, Bhatt HV. Long term exposure to Indian pan masala plain (PMP) and blend (PMT) causes gastro-hepatic injury in swiss albino mice. XII International Euroasian Congress of Surgery and Gastroenterology, (A), p138, Section: Diseases of Liver, Bile Ducts and Pancreas, Baki, 13-16, Oktyabr 2011, Baku: Azerbaijan Republic.

9. Cimolai N, Cimolai T. Svere iron deficiency anemia and gastrointestinal dysfunction associated with ingestion of pan masala. J Diet Suppl 2008;5:305-09.

10. Nigam SK, Bhatt HV. Dominant lethal studies in mice exposed to blended indian pan masala. Annals of National Academy of Medical Sciences India 2004:40:91-96.

11. Nigam SK, Bhatt HV. Effects of plain and blended indian pan masala on murine liver. Euroasian J Hepatogastoenterol 2011:1:27-29.

12. Nigam SK, Bhatt HV. Effects of Indian pan masala (plain and blended - PMP \& PMT) on testis after long-term feeding (PO) in mice. Int J Appl Biol pharmaec Tech 2012;3:235-41.

13. Rajendran R. Oral sub-mucous fibrosis: Etiology, pathogenesis and future research bull. WHO 1994;72,856-996.

14. Bhisey RA, Ramchandani AG, D'Souza AV, Borges AM, Notani PM. Long term carcinogenicity of pan masala in Swiss mice. Int J Cancer 1999;83:679-84.

15. Nigam SK, Karnik AB, Lakkad BC, Bhatt HV. Distribution of isomers of benzene hexachloride (BHC) and related histopathology of liver in hexachlorocyclohexane (HCCH) fed mice. Arch Env Hlth 1982;37:156-58.

16. Nigam SK, Bhatt HV. Biochemical effects of chronic plain and blended indian pan masala (PM) on liver in mice 99th Proceedings of Indian Science Congress, KIIT University, Bhubhaeshwar: Abstract, Jan 3-7, 2012.

17. Nigam SK, Bhatt HV. Effect of immunosuppressive agents on tumorogenesis in Swiss mice. Asian Med J 1982:25:809-15.

18. Klassen CD, Plaa GL. Relative effect of various chlorinated hydrocarbons on liver and kidney function in mice. Toxicol Appl Pharmacol 1966;9:139.

19. Idris AM, Nair J, Ohshima H, Friesen M, Brouet I, Faustman EM, Bartsch H. Unusually high levels of carcinogenic tobaccospecific nitrosamines in Sudan snuff (toombak). Carcinogenesis 199;12:1115-18.

20. Stich HF, Bohm B, Chatterjee K, Sails J. The role of saliva borne mutagens and carcinogens in etiology of oral and esophageal carcinomas of betel nut and tobacco chewers. Carcinogens and Mutagens in the Environment (Vol. II). In: Stich HF, CRC Press, Boca Raton FL (Eds). Naturally Occurring Compounds 1983:44-58.

21.. Ramachandani AG, DSouza AV, Berges AM, Bhisey RA. Evaluation of carcinogenic/cocarcinogenic activity of a common chewing product of pan masala in mouse skin, stomach and esophagus. Int J Cancer 1998:75:225-32.

22. Sarma AB, Chakrabarti J, Chakrabarti A. Evaluation of pan masala for toxic effects on liver and other organs. Food Chem Toxicol 1992:30:161-63.

\section{ABOUT THE AUTHORS}

\section{Suresh Kumar Nigam}

ICMR Emeritus Scientist, ES's Laboratory and Department of Neurobehavioral Toxicology, National Institute of Occupational Health (NIOH), Ahmedabad, Gujarat, India

\section{Huthi Venkatakrishna Bhatt (Corresponding Author)}

ICMR Emeritus Scientist, Ex-Senior Grade Deputy Director, Consulting Editor, ENVIS-NIOH Newsletter, ES'S Laboratory and Department of Neurobehavioral Toxicology, National Institute of Occupational Health (NIOH), Ahmedabad, Gujarat, India, e-mail: hvkbhatt@yahoo.co.in 